\title{
Supraphysiologic L-Tryptophan Elicits Cytoskeletal and Macromolecular Permeability Alterations in Hamster Small Intestinal Epithelium In Vitro
}

\author{
James L. Madara and Susan Carlson \\ Division of Gastrointestinal Pathology, Departments of Pathology, Brigham and Women's Hospital and Harvard Medical School; \\ and the Harvard Digestive Diseases Center, Boston, Massachusetts 02115
}

\begin{abstract}
We have previously shown that $\mathrm{Na}^{+}$-coupled transport of glucose and amino acids across the apical membrane of intestinal absorptive cells is accompanied by alterations in cytoskeletal structure and altered sieving of small hydrophilic solutes by tight junctions. Here we report that in response to the essential amino acid L-tryptophan at lumenal concentrations likely to be supraphysiological (1 $\mathrm{mM}$ or greater), these responses are so exaggerated as to induce disruption of tight junctions and transepithelial macromolecular leaks. Since these effects of L-tryptophan are energy and $\mathrm{Na}^{+}$dependent and occur with mucosal but not serosal exposure to L-tryptophan, it appears they are triggered by'activation of a $\mathrm{Na}^{+}$-nutrient cotransporter in the apical membrane of absorptive cells rather than by the presence of an unidentified trace contaminant. Our findings suggest the possibility that dietary supplementation by L-tryptophan may result in loss of the intestinal epithelial barrier to dietary antigens. We speculate that such a response to supraphysiologic tryptophan may contribute, in part, to the recently recognized curious tryptophan-induced eosinophilia myalgia syndrome. (J. Clin. Invest. 1991. 87:454-462.) Key words: eosinophilia myalgia syndrome - amino acid transport $\bullet$ microfilaments
\end{abstract}

\section{Introduction}

The major pathway for passive permeation across the epithelial lining of the small intestine is around cells, that is, paracellular $(1,2)$. The rate-limiting barrier within the paracellular pathway appears to be the intercellular tight or occluding junction that circumferentially wraps the apex of each epithelial cell (1). The tight junction is not static in its barrier function, however. Rather, substantial changes in tight junction ion and solute permeability can be elicited not only by a variety of factors present in disease states (3-5) but also by nonphysiologically induced alterations in intracellular mediators such as $\mathrm{Ca}^{++}$, cAMP, and protein kinase $\mathrm{C}$ (see above reviews). Physiological stimuli can also elicit altered junctional permeability (6-8). For example, exposure of the small intestine to lumenal glucose as would occur during a meal elicits increased tight junction permeability and altered tight junction structure (6), triggered by

Address reprint requests to Dr. James L. Madara, Department of Pathology, Brigham \& Women's Hospital, 20 Shattuck Street, Thorn Building, Room 1423, Boston, MA 02115.

Received for publication 23 July 1990 and in revised form 27 September 1990 .

J. Clin. Invest.

(c) The American Society for Clinical Investigation, Inc.

$0021-9738 / 91 / 02 / 0454 / 09 \$ 2.00$

Volume 87, February 1991, 454-462 turnover of the $\mathrm{Na}^{+}$-glucose cotransporters present within the apical plasma membrane of absorptive cells (7). Such reversible physiological regulation of tight junctions is thought to aid in absorption of lumenal nutrients by solvent drag (9) across junctions of enhanced permeability. Regulation of tight junction permeability also occurs in response to turnover of other $\mathrm{Na}^{+}$nutrient cotransporters within the absorptive cell apical membrane such as those for amino acids $(6,7)$. The increase in junctional permeability elicited by physiological substances, such as glucose, extends to small oligopeptides (10). However, as would be expected for a physiological state, glucose exposure does not induce loss of cell-cell contact (6), disintegration of cell junctions (6), or permeation of junctions by macromolecules (10).

Recently, while examining the physiological regulation of intestinal tight junctions by turnover of $\mathrm{Na}^{+}$-nutrient cotransporters in vitro we realized that the amino acid L-tryptophan elicits, when present in the mucosal bath at concentrations of 1-10 mM, a highly exaggerated response of the absorptive cell cytoskeleton with a parallel highly exaggerated increase in tight junction permeability. Such lumenal concentrations of L-tryptophan would likely only be encountered if excess L-tryptophan were ingested as a dietary supplement (see Discussion). Here we report these findings and raise the speculative hypothesis that L-tryptophan-induced breakdown of the intestinal epithelial barrier to dietary macromolecules might contribute to the recently recognized L-tryptophan-induced eosinophilia myalgia syndrome $(11-14)$.

\section{Methods}

Animals. Male golden hamsters weighing 180-220 $\mathrm{g}$ were anesthetized with pentobarbital sodium (Nembutal) at $5 \mathrm{mg} / 100 \mathrm{~g}$ body wt. The small intestine was removed, washed with ice-cold Ringer's solution, and opened longitudinally while on a $4^{\circ} \mathrm{C}$ surface. Samples were then stripped of muscularis propria and mounted into Ussing chambers as previously described (7). Briefly, the chambers were interfaced with two calomel electrodes and two $\mathrm{Ag}-\mathrm{AgCl}$ electrodes connected to the chamber by agar bridges. Both mucosal and serosal sides of the chamber were attached to circulating $10-\mathrm{ml}$ reservoirs equilibrated with $95 \% \mathrm{O}_{2} / 5 \% \mathrm{CO}_{2}$, and maintained at $37^{\circ} \mathrm{C}$. The circulating buffer solution consisted of (in mM) $114 \mathrm{NaCl}, 5 \mathrm{KCl}, 1.65 \mathrm{Na}_{2} \mathrm{HPO}_{4}, 0.3$ $\mathrm{NaH}_{2} \mathrm{PO}_{4}, 25 \mathrm{NaHCO}_{3}, 1.25 \mathrm{CaCl}_{2}$, and $1.1 \mathrm{MgSO}_{4}, \mathrm{pH}$ 7.4. The mucosal reservoirs contained the high oxygen affinity perfluorochemical Oxypherol-E.T. (20\% wt/vol; Alpha Therapeutic Corp., Los Angeles, CA). After a 15-min equilibration period, baseline resistance was determined. Baseline tissue resistance was recorded as the total minus the resistance of the bathing solution. Resistances were obtained using Ohm's law by measuring the voltage deflection elicited by $100 \mu \mathrm{A}$ of current (7). When solutes were added to one reservoir, they were osmotically balanced by the addition of an inert solute (mannitol or L-glucose) to the opposite reservoir. Transepithelial flux measurements were performed under short-circuited conditions as previously described (7). 


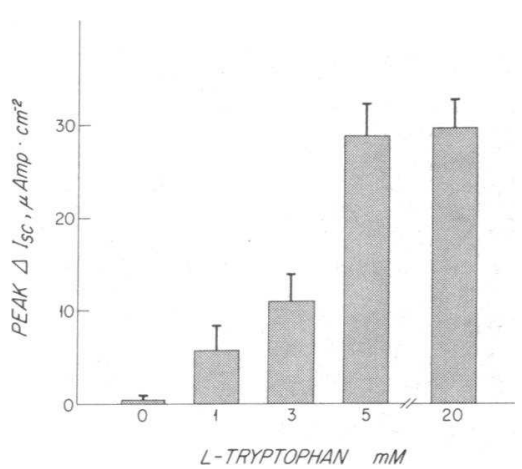

Figure 1. Electrical response of hamster small intestine to supraphysiologic concentrations of $\mathrm{L}$ tryptophan. Although 1-5-mM concentrations of L-tryptophan probably do not occur in the small intestinal lumen without dietary supplementation (see Discussion), this range does induce an

absorptive response as judged by $I_{\mathrm{sc}} . N$ for data points in this and subsequent figures $=6-14$.

Morphologic studies. Fixation of tissues for epoxy embedment was accomplished by addition of glutaraldehyde to the reservoirs to a final concentration of $2.5 \%$ (in the aforementioned Ringer's solution) (7). This allows one to maintain the electrical characteristics of the epithelium (6). Subsequent processing for routine thin and thick section microscopy was as previously described $(6,7)$. Fluorescence localization of actin was carried out as before (5) and intermediate filaments were identified by a cytokeratin antibody (mAb CaCo $3 / 28$, kindly provided by Dr. A. Quaroni, Cornell University). For the latter stain, sections were blocked with goat serum at a 1:25 dilution in PBS and washed three times in gel-PBS before the 1-h staining period at $37^{\circ} \mathrm{C}$. After three additional gel-PBS washes, sections were incubated with rhodamine-conjugated goat anti-mouse IgG, washed in gel-PBS, and mounted in glycerol-PBS- $p$-phenylenediamine. Use of and localization of horseradish peroxidase (HRP) ${ }^{1}$ was as previously described (15).

Statistics. Comparisons were made using unpaired Student's $t$ test.

\section{Results}

L-tryptophan elicited electrical responses typical of $\mathrm{Na}^{+}$-cotransported nutrients. Mucosal addition of L-tryptophan induced an increase in short circuit current $\left(I_{\mathrm{sc}}\right)$ (Fig. 1) that was maximal at $5 \mathrm{mM}\left(29 \pm 3,11 \pm 3\right.$, and $6 \pm 3 \mu \mathrm{A} \cdot \mathrm{cm}^{-2}$ for 5,3 , and $1 \mathrm{mM}$, respectively). The $I_{\mathrm{sc}}$ response elicited by $5 \mathrm{mM}$ L-tryptophan was $82 \%$ reduced $(P<0.01)$ in tissues preexposed for $15 \mathrm{~min}$ to $0.25 \mathrm{mM} \mathrm{DNP}$ (Fig. 2), a concentration that we have previously shown (16) does not affect resistance but depletes energy stores by $70-80 \%$ as defined functionally by a reduction in spontaneous $I_{\text {sc }}$. The $5 \mathrm{mM}$ L-tryptophan response was ablated $(P<0.005)$ in experiments in which mucosal $\mathrm{Na}^{+}$was replaced with choline (Fig. 2). Thus the L-tryptophan-induced $I_{\mathrm{sc}}$ is energy and $\mathrm{Na}^{+}$dependent. L-tryptophan exposure also resulted in a dose-dependent decrease in transepithelial resistance (Fig. 3). At a dose of $5 \mathrm{mM}$, which is saturating according to the $I_{\mathrm{sc}}$ response (Fig. 1), resistance fell $14 \pm 3$ ohms $\cdot \mathrm{cm}^{2}(P<0.01)$ within 5 min of mucosal addition and maintained this value over the ensuing 20 -min period (Fig. 3). The $I_{\mathrm{sc}}$ and resistance responses elicited by mucosal L-tryptophan were not elicited by serosal exposure of $L$-tryptophan $\left(2 \pm 1 \mu \mathrm{A} \cdot \mathrm{cm}^{-2}\right.$ and $3 \pm 2 \mathrm{ohm} \cdot \mathrm{cm}^{2}$ for change in $I_{\mathrm{sc}}$ and resistance, from baseline, respectively, $P=\mathrm{NS}$ ).

$L$-tryptophan elicited responses unusual of $\mathrm{Na}^{+}$-cotransported nutrients. The $I_{\mathrm{sc}}$ response elicited by mucosal addition

1. Abbreviations used in this paper: HRP, horseradish peroxidase; $I_{\mathrm{sc}}$, short circuit current.

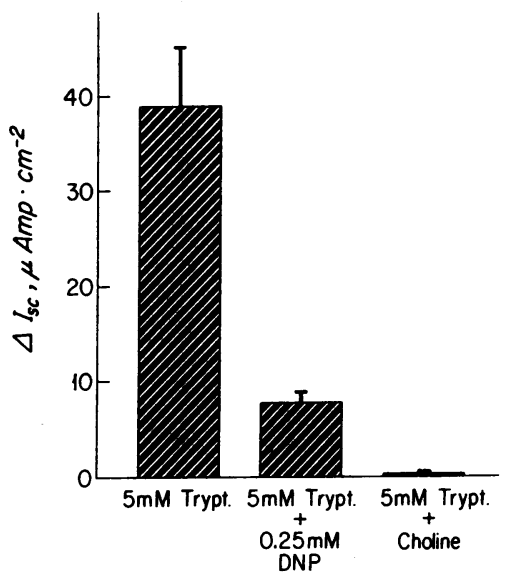
by $5 \mathrm{mM}$ mucosal Ltryptophan is energy and $\mathrm{Na}^{+}$dependent as would be expected for a $\mathrm{Na}^{+}$-cotransported nutrient. DNP, dinitrophenol.
Figure 2. The $I_{\mathrm{sc}}$ elicited

of and subsequent continuous exposure to L-tryptophan was transient at concentrations of $1 \mathrm{mM}$ or greater (Fig. 4) (including concentrations from 10 to $20 \mathrm{mM}$, data not shown). After addition of $5 \mathrm{mM}$ mucosal L-tryptophan, $I_{\mathrm{sc}}$ peaked at $2.7 \mathrm{~min}$ but immediately declined to reach baseline values by $12 \mathrm{~min}$. By 30 min (Fig. 4), despite the continuing presence of mucosal L-tryptophan, $I_{s c}$ was no different than that in tissues unexposed to mucosal L-tryptophan (Fig. 4). The short-lived $I_{\text {sc }}$ response to L-tryptophan contrasts with that elicited by other $\mathrm{Na}^{+}$-cotransported nutrients including glucose (Fig. 5) or amino acids as represented by $\mathrm{L}$-proline, $\mathrm{L}$-methionine, or $\mathrm{L}$ phenylalanine (Fig. 6). A saturating concentration of glucose $(25 \mathrm{mM})$ resulted in a steady-state $I_{\mathrm{sc}} \sim 200 \mu \mathrm{A} \cdot \mathrm{cm}^{-2}$ above baseline that was maintained through $30 \mathrm{~min}$ (Fig. 5). Similarly, mucosal exposure to $5 \mathrm{mM}$ of L-proline, L-methionine, or L-phenylalanine resulted in $30-\mathrm{min} I_{\mathrm{sc}}$ values substantially greater than baseline $\left(55 \pm 7,47 \pm 8\right.$, and $38 \pm 6 \mu \mathrm{A} \cdot \mathrm{cm}^{-2}$, respectively) which contrasted with the $5 \mathrm{mM}$ L-tryptophan response $\left(17 \pm 6 \mu \mathrm{A} \cdot \mathrm{cm}^{-2}\right.$ below baseline; $P<0.01$ compared with glucose, L-proline, L-methionine, or L-phenylalanine). Also in contrast to $5 \mathrm{mM}$ L-proline, L-methionine, or L-phenylalanine, $5 \mathrm{mM}$ L-tryptophan yielded a substantially lower peak $I_{\mathrm{sc}}$ $\left(57 \pm 8,53 \pm 4\right.$, and $38 \pm 6$ vs. $25 \pm 6 \mu \mathrm{A} \cdot \mathrm{cm}^{-2}$ for L-proline, Lmethionine, and L-phenylalanine vs. L-tryptophan; all $P$ $<0.01$, Fig. 6). As shown in Fig. 7, tissues preexposed to $5 \mathrm{mM}$ L-tryptophan for $15 \mathrm{~min}$, washed, and then exposed to $25 \mathrm{mM}$ glucose displayed a glucose-elicited $I_{\mathrm{sc}} 45 \pm 12 \mu \mathrm{A} \cdot \mathrm{cm}^{-2}$ less than tissues identically treated but not preexposed to L-tryptophan $(P<0.01)$. We also found, as has been suggested by others

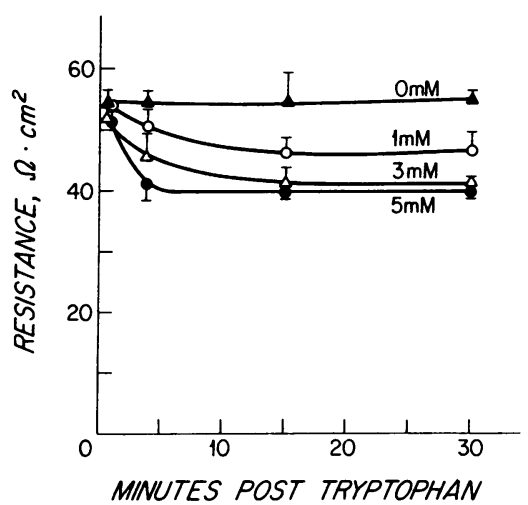

Figure 3. 1-5 mM Ltryptophan elicits a decrease in transepithelial resistance to passive ion flow. 


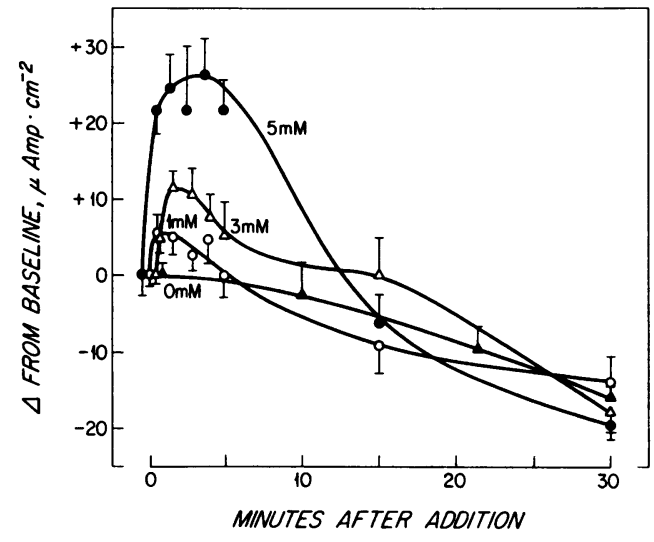

Figure 4. Time course of $I_{\mathrm{sc}}$ response to L-tryptophan. The $I_{\mathrm{sc}}$ response to mucosal L-tryptophan at $1-5 \mathrm{mM}$ concentrations peaks within $5 \mathrm{~min}$ and then diminishes to baseline values. Similar transient $I_{s c}$ peaks are also seen at concentrations from 10 to $20 \mathrm{mM}$ (not shown).

(see Discussion), that millimolar concentrations of L-tryptophan could suppress the subsequent response to other $\mathrm{Na}^{+}$cotransported nutrients, such as L-proline, as well (data not shown).

Effect of $M M$ L-tryptophan on general epithelial structure. Mucosal L-tryptophan exposure elicited general alterations in epithelial structure predominantly localized to the villus tips and not seen with other $\mathrm{Na}^{+}$-cotransported nutrients, such as
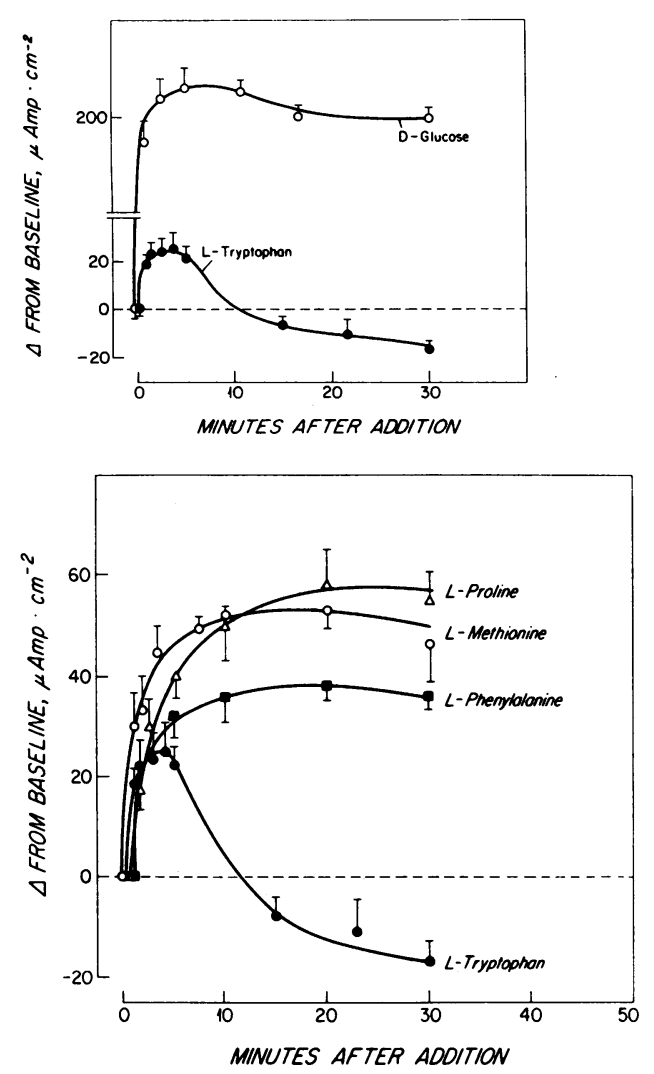

Figures 5 and $6 . I_{\mathrm{sc}}$ response to L-tryptophan compared with other $\mathrm{Na}^{+}$-cotransported nutrients. In contrast to that elicited by glucose (Fig. 5) or other amino acids such as L-proline, L-methionine, or L-phenylalanine (Fig. 6), the $I_{\mathrm{sc}}$ response to L-tryptophan is short-lived.

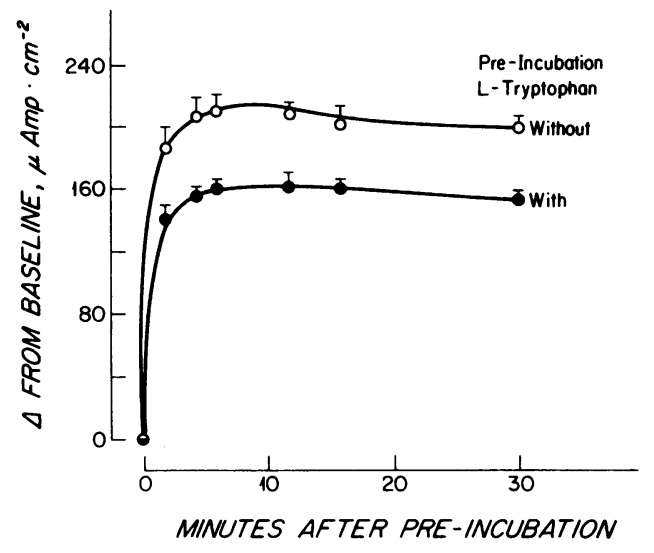

Figure 7. Effect of L-tryptophan preexposure ( $15 \mathrm{~min}, 5 \mathrm{mM}$ followed by washout) on subsequent $I_{\mathrm{sc}}$ response to mucosal glucose (20 mM). The preincubation with L-tryptophan has an antiabsorptive effect as judged by diminution of the glucose-elicited $I_{\mathrm{sc}}$ response $(P<0.01)$.

glucose. As shown in the 1- $\mu \mathrm{m}$ sections displayed in Fig. 8, after 15-30 min exposure to $5 \mathrm{mM} \mathrm{L}$-tryptophan absorptive cells at the villus tip tended to lift from the basement membrane, remaining attached to the matrix by thin cytoplasmic projections. This finding does not occur subsequent to exposure to $\mathrm{Na}^{+}$-cotransported nutrients, such as glucose and L-leucine (6). The apex of the absorptive cells also showed rounding (Fig. 8) comparable to the rounding reported with cytoskeletal contraction in isolated brush borders $(17,18)$ or brush border rounding induced by nonphysiological means in whole absorptive cells $(16,19)$. These changes were first seen $15 \mathrm{~min}$ after exposure to 3-5 $\mathrm{mM}$ L-tryptophan and $30 \mathrm{~min}$ after exposure to 1 mM L-tryptophan. Although subtle rounding of the brush border, barely detectable by light microscopy, can focally be seen with exposure to glucose or L-leucine (6), the obvious rounding as well as the deformation of the basal poles of absorptive cells, seen with L-tryptophan, is not.

Effects of L-tryptophan on absorptive cell tight junctions. As previously reported in detail $(6,7)$, tissues exposed to D-glucose or an L-amino acid other than L-tryptophan exhibit, by transmission electron microscopy, tight junctions that focally contain distortions in the form of dilatations, but are otherwise
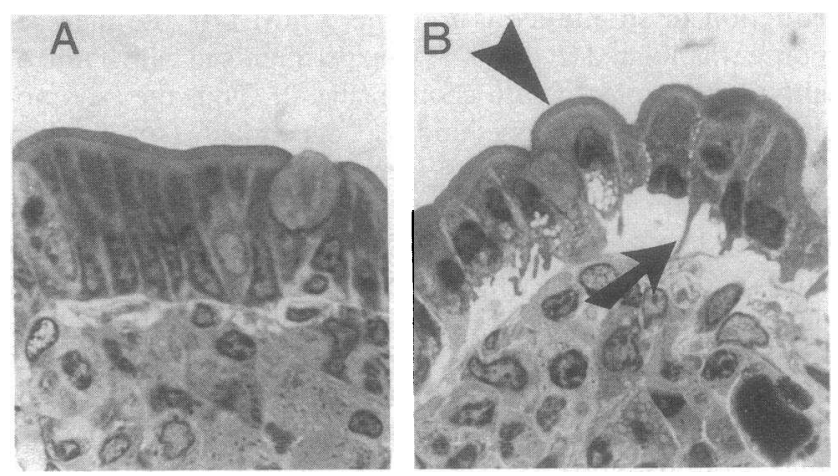

Figure 8. Light micrographs of chamber-mounted intestinal villus-tip epithelium in the control state $(A)$ or after exposure to $5 \mathrm{mM}$ mucosal L-tryptophan $(B)$. After L-tryptophan exposure the apex of absorptive cells is obviously rounded (arrowheads) and cells separate from the basement membrane, being attached only by thin cytoplasmic projections (arrow). 


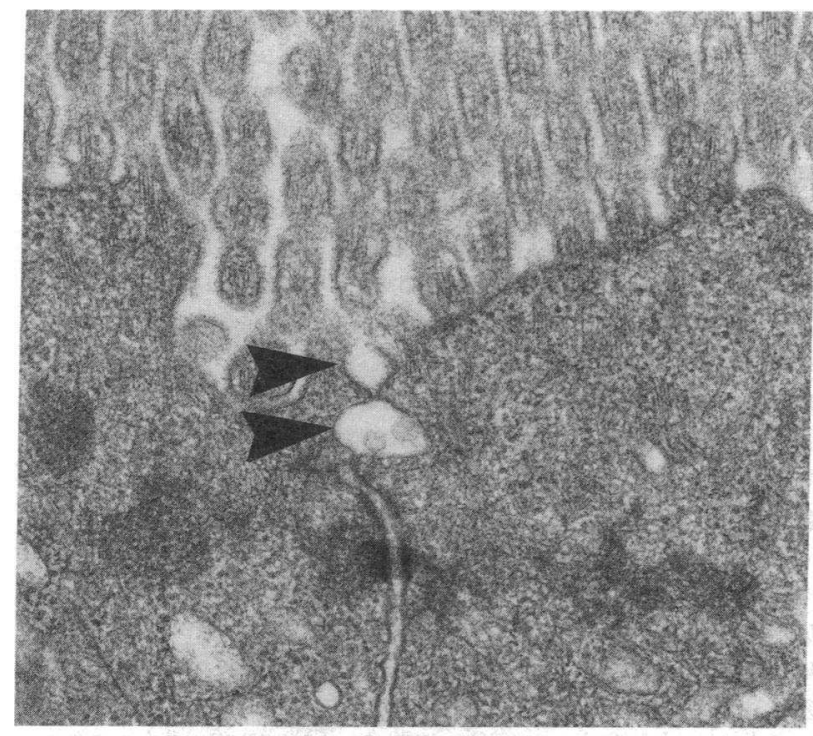

Figure 9. As we have previously reported for other $\mathrm{Na}^{+}$-cotransported nutrients $(6,7)$, mucosal exposure to L-tryptophan elicited tight junction dilatations (arrowheads).

normal. Such tight junction dilatations are also elicited by Ltryptophan (Fig. 9) but their frequency was substantially attenuated in tissues in which mucosal $\mathrm{Na}^{+}$was replaced by choline (e.g., are $\mathrm{Na}^{+}$dependent as we have shown for the dilatations induced by other $\mathrm{Na}^{+}$nutrient cotransport systems, 7). However, in contrast to D-glucose or the other L-amino acids studied, mucosal L-tryptophan exposure also induced other, more marked, perturbations in tight junctions, focally including apparent complete disruption. As seen in Fig. $10 \mathrm{~B}, 30 \mathrm{~min}$ expo- sure to even $1 \mathrm{mM}$ L-tryptophan focally resulted in images in which absorptive cells had apparently separated from their neighbors at the level of the tight junction. More extreme examples of such extensive separation of cells at tight junctions could be seen in tissues exposed to $5 \mathrm{mM}$ L-tryptophan for 30 min (Fig. $10 \mathrm{C}$ ). Given proposed tight junction structure-function correlates (1) the above findings suggested that junctional permeability might be markedly disordered after L-tryptophan exposure. Flux studies showed that a 20 -min exposure to $5 \mathrm{mM}$ L-tryptophan elicited a $296 \pm 21 \%$ increase in transepithelial inulin flux in the ensuing 10-min flux period as compared with matched controls ( $5 \mathrm{mM}$ mannitol; $P<0.01$ ). Although other $\mathrm{Na}^{+}$-cotransported nutrients, such as glucose, also increase junctional permeation to hydrophilic solutes (9), in in vitro preparations such increases, although demonstrable (7), are markedly more modest $(<35 \%)$ even when comparing smaller solutes such as mannitol. In data not shown, we verified this by showing that mean inulin flux in tissues exposed to $20 \mathrm{mM}$ glucose was only $16 \%$ greater than mean inulin flux in control tissues ( $5 \mathrm{mM}$ mannitol) compared with the $300 \%$ increase in inulin flux elicited by L-tryptophan. Enhanced junctional permeability elicited by L-tryptophan extended to macromolecules as well. As shown in Fig. 11, L-tryptophan exposure (5 $\mathrm{mM}, 30 \mathrm{~min}$ ) in the presence of $0.5 \%$ mucosal HRP elicited readily apparent transepithelial leaks of this macromolecule. Such macromolecular leaks were present in $68 \%$ of villi, under these conditions. Control experiments verified, as previously shown $(10,15)$ that comparable HRP leaks do not occur in tissues even when exposed to D-glucose.

Effects of L-tryptophan on the absorptive cell cytoskeleton. Within $5 \mathrm{~min}$ of exposure to 1-5 mM L-tryptophan, spicules of condensed cytoskeletal elements with features suggestive of intermediate filaments appeared in absorptive cells (Fig. 12).
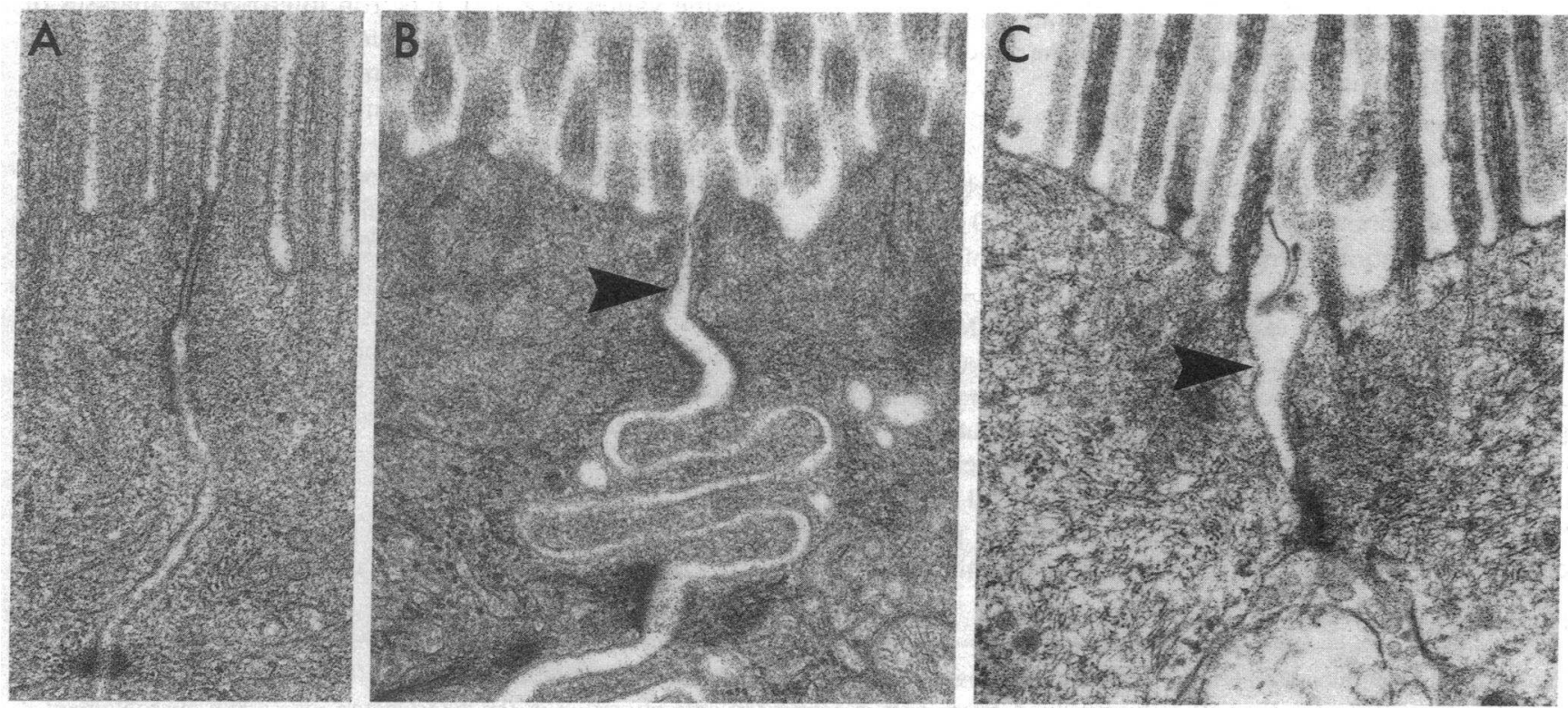

Figure 10. Electron micrographs of villous absorptive cells. (A) After mucosal exposure to $20 \mathrm{mM}$ glucose (control) or after mucosal exposure to $1(B)$ or $5 \mathrm{mM}(C)$ L-tryptophan for $30 \mathrm{~min}$. For purposes of comparison, an example of the appearance of tight junctions, after glucose exposure, in areas without dilatations is shown in $A$. The only glucose-elicited changes in tight junctions that occur are dilatations like those induced by L-tryptophan and shown in Fig. 9 as we have previously reported in great detail $(6,7)$. Unlike the effects of glucose on tight junction structure, L-tryptophan elicits changes in which the close membrane-membrane apposition between cells is focally lost (arrowheads) thus suggesting a grossly opened tight junction. 


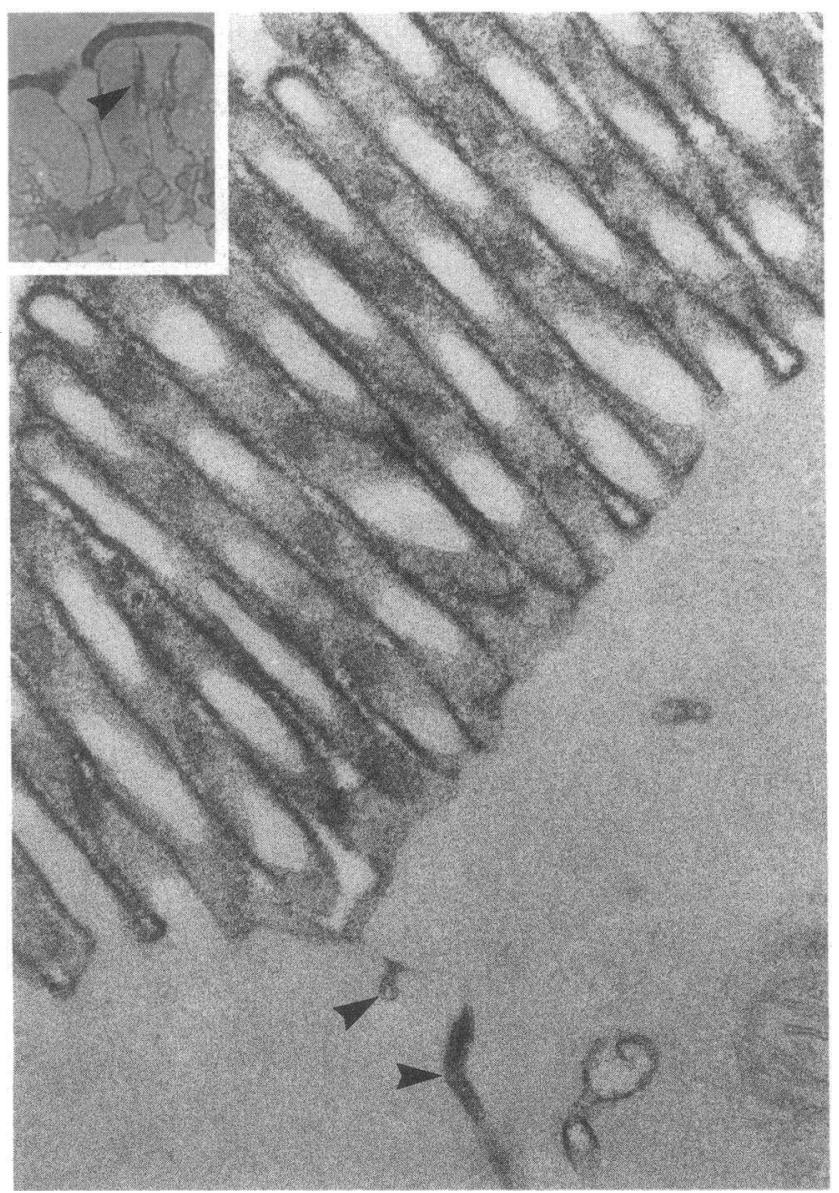

Figure 11. Micrographs of tissues exposed to 20-30 min mucosal Ltryptophan $(5 \mathrm{mM})$ in the presence of the macromolecular marker HRP in the mucosal reservoir. (Inset) Light micrograph showing linear penetration of the macromolecule across the epithelium (arrowhead). (Main panel) Unstained electron micrograph highlighting the dense accumulation of tracer in the paracellular space underlying the tight junction (arrowheads).

These were primarily seen across the terminal web and along the lateral membrane (Fig. 12). As shown in Fig. $10 \mathrm{C}$, microfilament condensations were also seen in the area of tight junctions that were markedly altered structurally by L-tryptophan exposure. After $30 \mathrm{~min}$ exposure to higher $(20 \mathrm{mM})$ L-tryptophan concentrations (Fig. $12 \mathrm{C}$ ), the perijunctional cytoskeleton focally appeared detached from the lateral membrane and aggregated in two forms: as plaques of intermediate filamentlike elements, and as aggregates of microfilament-like elements. In areas in which the cytoskeleton was still attached to the lateral membrane, the intermediate filament plaques described above existed although the microfilament aggregates were less clearly delineated and consisted of thickened terminal web areas that rounded the cell apex. Also, under these latter conditions, vesiculation of intracellular membrane compartments occurred. Lastly, aggregation of cytoskeletal elements were apparent in the base of villous tip absorptive cells.

Fluorescence localization of cytokeratin (e.g., intermediate filaments) and F-actin (e.g., microfilaments) confirmed the electron microscopic interpretations. As seen in Figs. 13 and 14, L-tryptophan exposure resulted in rounded brush borders and accumulation of F-actin at the base of the cell. L-tryptophan exposure also elicited a rearrangement of intermediate filaments from a fine reticular network into plaque-like condensations (again also with formation of basal plaques) (Fig. 14).

Structural responses to L-tryptophan appear to be dependent on L-tryptophan transport across the apical membrane of $a b$ sorptive cells. We also attempted to assess whether the above structural effects were related to L-tryptophan absorption vs. the presence of a potential unknown trace contaminant. First, we performed sidedness experiments. The profound morphologic responses to mucosal L-tryptophan, described above, were not reproduced by serosal L-tryptophan exposure (not shown) and, as previously stated, serosal L-tryptophan exposure did not elicit an $I_{s c}$ or resistance response. Second, with replacement of mucosal $\mathrm{Na}^{+}$by choline (conditions that prevent $\mathrm{Na}^{+}$-nutrient cotransport across the apical membrane; also see Fig. 2), the structural responses to L-tryptophan were attenuated. Thus, both the structural as well as the functional responses we described appear to be dependent on an active $\mathrm{Na}^{+}$-dependent uptake of $\mathrm{L}$-tryptophan from the mucosal reservoir.

Initiation of L-tryptophan absorption does not fully commit epithelium to L-tryptophan response. $5 \mathrm{~min}$ exposure to $5 \mathrm{mM}$ L-tryptophan elicits the full resistance response, but subsequent washout of L-tryptophan allows a return of resistance toward baseline values (Fig. 15). Electron microscopic analyses of these tissues revealed that focal intermediate filament spicules (as would be present at $5 \mathrm{~min}$ ) were maintained through $30 \mathrm{~min}$ and, thus, the early condensation of intermediate filaments is not reversible in this short time. However, the more marked alterations described above that occur 15-30 min after L-tryptophan exposure were not present in these tissues. Thus, full expression of the L-tryptophan effects appears to require continued activity of the L-tryptophan-elicited absorption response. Not surprisingly, the resistance decrease elicited by 30 min exposure to $5 \mathrm{mM}$ L-tryptophan was not reversible in the ensuing 40-min period (data not shown).

\section{Discussion}

We show that, like the other the $\mathrm{Na}^{+}$-cotransported nutrients $(6,7)$, L-tryptophan elicits a decrease in transepithelial resis-

Figure 12. Electron micrographs of absorptive cells following exposure to $20 \mathrm{mM}$ mucosal glucose for $30 \mathrm{~min}(A)$, or $1 \mathrm{mM}(B)$, or $20 \mathrm{mM}(C)$ mucosal L-tryptophan for $5^{\prime}$ or $30^{\prime}$, respectively. After glucose exposure the only alteration in the cytoskeleton seen is the microfilament condensation just below the tight junction in the area of the perijunctional actin-myosin ring $(6,7)$ (arrowheads). A glucose-induced dilatation in the tight junction is present. In contrast, after only a 5-min exposure to $1 \mathrm{mM}$ L-tryptophan, even in areas not containing junctional dilatations, dense plaques of cytoskeletal elements are seen across the terminal web and along the lateral membrane. These plaques appear to be composed of intermediate filaments. In the extreme $(C)$, cytoskeletal elements could be seen completely pulling away from the lateral membrane at the site of the junctional complex (bracket). Both microfilament aggregates (arrows) and intermediate filament aggregates, packed in paracrystalline array (arrowheads), are seen within the terminal web. 


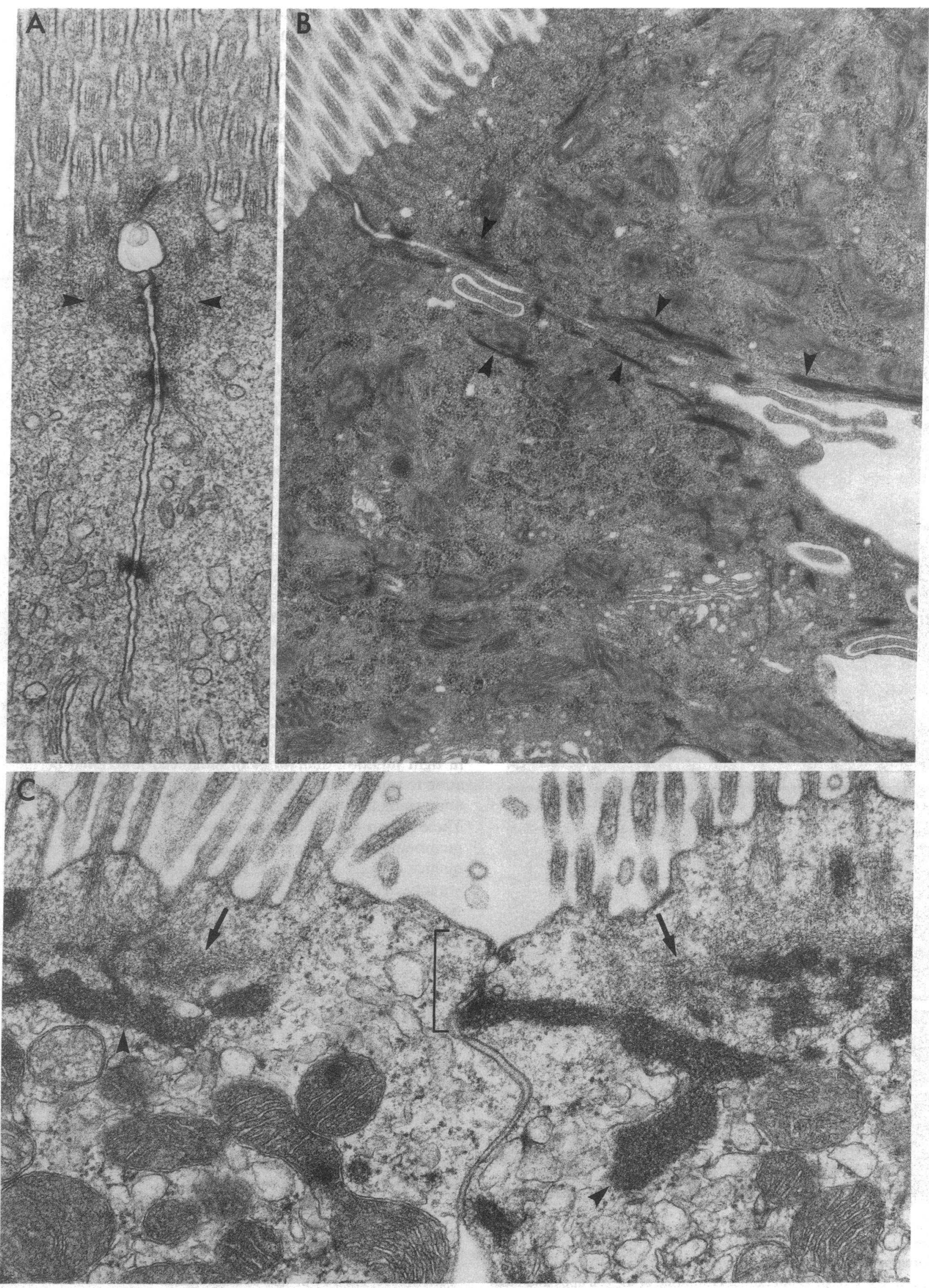



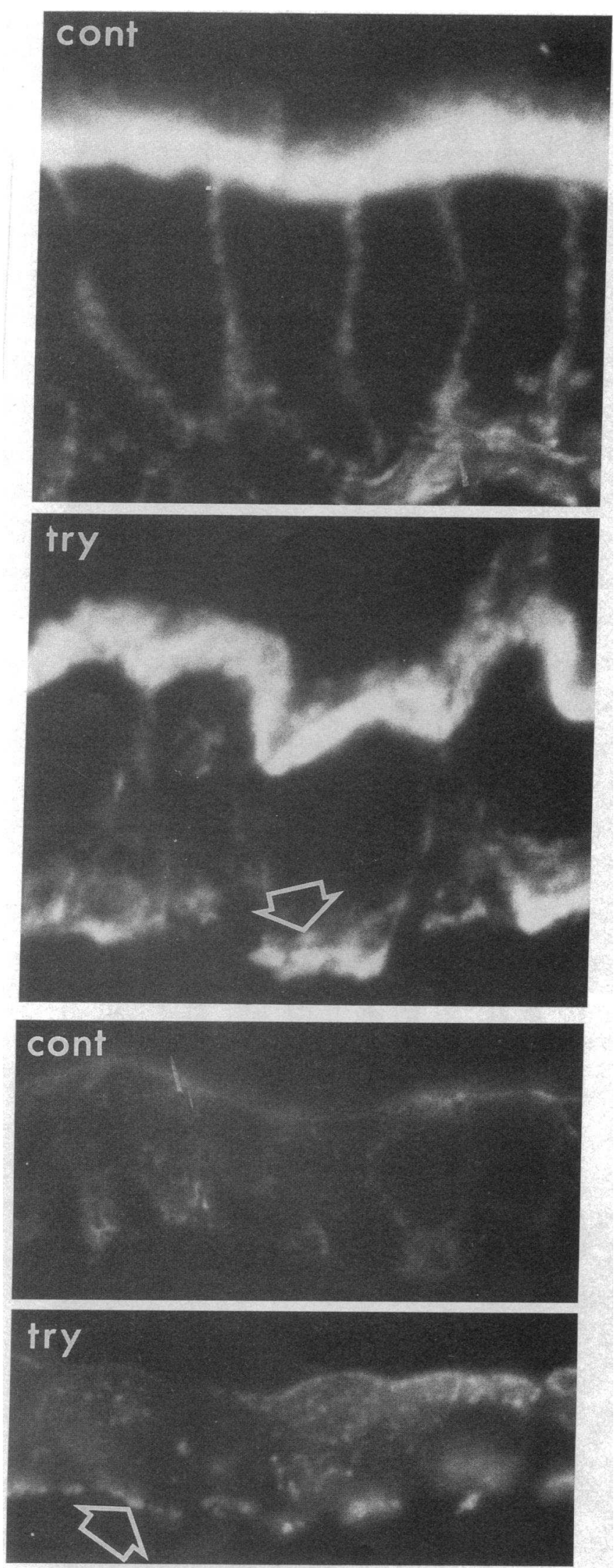

Figures 13 and 14. Fluorescence micrographs of control and Ltryptophan ( $5 \mathrm{mM}, 30 \mathrm{~min}$ ) exposed villus tip epithelium. Micrographs show F-actin (13) and cytokeratin (14) localization, respectively. L-tryptophan induces rounding of the cell apex and basal tance accompanied by alterations in tight junction structure. As expected, L-tryptophan also elicits an increase in $I_{\mathrm{sc}}$ that is $\mathrm{Na}^{+}$and energy dependent as do other $\mathrm{Na}^{+}$-cotransported nutrients. Surprisingly, however, L-tryptophan exposure results in an array of alterations that differ from those following exposure of the intestine to other $\mathrm{Na}^{+}$-cotransported nutrients studied: the $I_{\mathrm{sc}}$ response to L-tryptophan is short lived; L-tryptophan elicits focal complete disruption of tight junction structure and macromolecular barrier function; and L-tryptophan elicits profound alterations in the cytoskeleton including the intermediate filament network. We do not know if changes in intermediate filaments could alter junctional permeability; these filaments are thought to largely interact with desmosomes. One possibility is that loss of desmosomal stabilization countering baseline tension in the perijunctional actomyosin ring could effectively enhance lateral tension in the area of the junctional complex.

Although L-tryptophan transport has not been nearly as well characterized as that of many other amino acids, previous work in this area does suggest that curiosities do exist with transport of this particular amino acid. For example, Spencer and Samily (20), using everted sacs, showed that, while at low concentrations, L-tryptophan could accumulate (indicative of absorption); paradoxically, at higher concentrations accumulation diminished. Similarly, Munck and Rasmussen (21) noted that L-tryptophan appeared to accelerate a decline in transepithelial potential difference in rat jejunum and suggested that L-tryptophan might have a nonspecific inhibitory effect on epithelial transport functions of intestinal epithelial cells. These peculiar effects of L-tryptophan also extend to the newborn rabbit jejunum as nicely demonstrated by Cooke et al. $(22,23)$.

Our findings suggest that the basis for the above-outlined peculiar effects of L-tryptophan (at millimolar concentrations) may be an exaggeration of the normal responses of absorptive cells to $\mathrm{Na}^{+}$-cotransported nutrients. We have previously reported that $\mathrm{D}$-glucose, L-proline, and L-alanine exposure result in tight junction dilatations or enhanced junctional permeation to small solutes $(6,7)$ accompanied by subtle cytoskeletal alterations suggestive of enhanced cytoskeletal tension (6). These alterations are initially triggered by activation of apical membrane $\mathrm{Na}^{+}$-nutrient cotransporters (7) and, as a consequence of these alterations, paracellular nutrient absorption, via solvent drag, occurs and contributes to net nutrient absorption (9). However, the cytoskeletal alterations and associated brush border rounding (as occurs with nonphysiologically induced cytoskeletal contraction in brush borders $[17,18]$ or in intact cells [16]) that occur in response to L-tryptophan are markedly increased over those that occur in response to other $\mathrm{Na}^{+}$-cotransported nutrients that we have studied $(6,7)$. Moreover, the involvement of the intermediate filament network in the cytoskeletal response to $\mathrm{Na}^{+}$-nutrient cotransport has not been noted for other nutrients such as glucose and leucine. It is of interest that only recently has it been appreciated that the intermediate filament network of the cytoskeleton may be dynamic as is the microfilamentous network (24). For example, changes highly similar in phenotype to those we report in response to L-tryptophan occur with phosphorylation of inter-

accumulation of plaques of both F-actin and cytokeratin (arrowheads). In addition the normal lacy reticular cytokeratin pattern is replaced by plaque-like aggregates. 


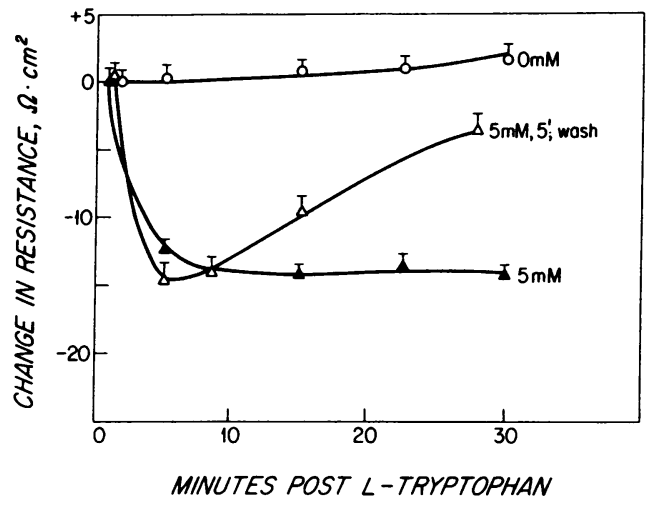

Figure 15. Resistance effect of 0 and $5 \mathrm{mM}$ mucosal L-tryptophan compared with $5^{\prime}$ exposure to L-tryptophan followed by an Ltryptophan washout (at $5 \mathrm{~min}$ ). Initiation alone of the L-tryptophan response is insufficient to elicit the full response (also as judged structurally; see text).

mediate filament subunits during physiological events such as movement through the cell cycle. It is of interest in the regard that phosphorylation events would also be expected to underlie the microfilament condensations that presumably are dependent on myosin-actin interactions (18).

The exaggerated cytoskeletal and/or junctional permeability responses of the epithelium to L-tryptophan seem to be linked, like the less vigorous responses to D-glucose, L-proline, and $L$-alanine, to movement of this nutrient across the apical membrane. The L-tryptophan responses are not elicited by basolateral addition or by apical addition in either the energy-depleted state or without mucosal $\mathrm{Na}^{+}$. Such findings strongly suggest that the observed effects are triggered by $\mathrm{Na}^{+}$-dependent L-tryptophan uptake across the apical membrane of absorptive cells. By inducing such dramatic effects on epithelial structure which are paralleled by loss of the epithelial barrier to macromolecular permeation and inhibition of other epithelial transport events such as glucose absorption, it is fair to say that 1-5 mM concentrations of mucosal L-tryptophan actually exhibit toxic effects on the intestinal epithelium. A recent morphological study reporting that intestinal tight junctions leak HRP in vivo in animals exposed to $20 \mathrm{mM}$ lumenal L-tryptophan demonstrates that these responses occur in intact animals as well as in isolated mucosal sheets (25). What biological advantage could possibly exist in such a response to a dietary nutrient? First, one must recognize, as outlined above, that the subtler cytoskeletal and tight junction permeability responses to other $\mathrm{Na}^{+}$-cotransported nutrients result in a physiologically advantageous paracellular absorption of nutrients. L-tryptophan is the essential nutrient (e.g., an essential amino acid) least prevalent in the diet (26). Thus, teleologically, it would make sense that the phenotypic response of absorptive cells to $\mathrm{Na}^{+}$-cotransported nutrients be most sensitively coupled to $\mathrm{L}-$ tryptophan, particularly if the L-tryptophan concentrations that elicit the exaggerated "toxic" responses are not encountered physiologically. Based on our studies and others in the literature (20-23) it would appear that such toxic effects of L-tryptophan begin at concentrations of $\sim 1-2.5 \mathrm{mM}$. To our knowledge, it is not known what the physiological L-tryptophan concentration in the intestinal lumen is. However, since the postprandial concentrations of several amino acids in the rat jejunum have been measured (27) and the dietary proportion of L-tryptophan to other amino acids is known (26), one can estimate physiologic lumenal L-tryptophan concentrations. The relative proportions of the various amino acids in the diet (26) is similar to the relative proportions of various amino acids in the rat jejunum postprandially, thus verifying such estimates as reasonable. Based on such analysis, one would estimate that the jejunal L-tryptophan concentrations on a physiological diet would be $<1 \mathrm{mM}$ (maximal "physiologic" L-tryptophan would be $\sim 0.7 \mathrm{mM}$, based on such an estimate), a concentration just below that apparently necessary for the inhibitory (toxic) effects of L-tryptophan on intestinal absorption (20-23). Thus, the grossly exaggerated responses reported here are likely not to occur with the ordinary diet.

Given the rather dramatic effects of supraphysiologic concentrations of L-tryptophan on epithelial structure and tight junction macromolecular permeability, it is of interest that dietary supplementation with gram quantities of L-tryptophan has been noted to produce a poorly defined systemic illness (11-14). This illness, recently termed the tryptophan-induced eosinophilia-myalgia syndrome (14), represents a diverse array of signs and symptoms that include not only the manifestations for which it is named but dyspnea, urticarial rashes, and fatigue. The pathogenesis of this syndrome, paradoxically induced by an essential nutrient, is opaque at this time. Since it was not expected that an essential amino acid should elicit such ill effects, recent hypothetical possibilities raised to explain the pathogenesis of this syndrome include the presence of a contaminant or a high prevalence inborn error in tryptophan metabolism (14). Recently published epidemiological evidence suggests that a contaminant in L-tryptophan preparations plays an important role in this syndrome (27). Based on this report, we raise the speculative possibility that the intestinal epithelial responses to supraphysiological tryptophan concentrations may be involved in the manifestations of this syndrome. Specifically, since based on our finding in vitro and other reports in vivo (25) that permeation of food proteins across the intestine should be expected with high lumenal L-tryptophan concentrations, it might not be surprising if patients ingesting gram quantities of L-tryptophan exhibited symptoms of chronic food intolerance or food allergy. Symptoms of these latter syndromes also include a diverse array of signs and symptoms including eosinophilia, fatigue, and urticaria (28). We emphasize that linking the tryptophan-induced eosinophilia-myalgia syndrome to the exaggerated intestinal epithelial responses of supraphysiologic L-tryptophan concentrations is mere speculation at this time. On the other hand, this new hypothesis should be testable and rests on the altogether surprising observation of intestinal epithelial-targeted toxicity triggered by $\mathrm{Na}^{+}$-tryptophan cotransport under conditions in which this essential amino acid is present at supraphysiologic concentrations in the lumen.

\section{Acknowledgments}

This work was supported by National Institutes of Health grant R01DK-35932.

\section{References}

1. Madara, J. L. 1989. Loosening tight junctions. Lessons from the intestine. J. Clin. Invest. 83:1089-1094. 

G288.

. Powell, D. 1981. Barrier function of epithelia. Am. J. Physiol. 241:G275-

3. Nash, S., J. Stafford, and J. L. Madara. 1987. Effects of polymorphonuclear leukocyte transmigration on the barrier function of cultured intestinal epithelial monolayers. J. Clin. Invest. 80:1104-1113.

4. Lopez-Vancell, R., G. Beatty, E. Stefani, E. E. Rodriguez Boulan, and M. Cereijido. 1984. Changes in paracellular ionic permeabilities of monolayers of MDCK cells infected with influenza or vesicular stomatitis virus. J. Membr. Biol. 81:171-180.

5. Madara, J. L., and J. Stafford. 1989. Interferon- $\gamma$ directly affects barrier function of cultured intestinal epithelial monolayers. J. Clin. Invest. 83:724-727.

6. Madara, J. L., and J. R. Pappenheimer. 1987. Structural basis for physiological regulation of paracellular pathways in intestinal epithelia. J. Membr. Biol. 100:149-164.

7. Atisook, K., S. Carlson, and J. L. Madara. 1990. Effects of phlorizin and sodium on glucose-elicited alterations of cell junctions in intestinal epithelia. Am. J. Physiol. 258:C77-C85.

8. McRoberts, J. A., R. Aranda, N. Riley, and H. Kang. 1990. Insulin regulates the paracellular pathway of cultured intestinal epithelial cell monolayers. $J$. Clin. Invest. 85:1127-1134.

9. Pappenheimer, J. R., and K. Z. Reiss. 1987. Contribution of solvent drag through intercellular junctions to absorption of nutrient by the small intestine of the rat. J. Membr. Biol. 100:123-136.

10. Atisook, K., S. Carlson, and J. L. Madara. An oligopeptide permeates intestinal tight junctions at glucose-elicited dilatations: implications for oligopeptide absorption. Gastroenterology. In press.

11. Eosinophilia-myalgia syndrome-New Mexico. 1989. Morbidity Mortality Weekly Report (MMWR). 38:765-767.

12. Hertzman, P. A., W. L. Blevins, J. Mayer, B. Greenfield, M. Ting, and G. J. Gleich. 1990. Association of the eosinophilia-myalgia syndrome with the ingestion of tryptophane. N. Engl. J. Med. 322:869-873.

13. Silver, R. M., M. P. Heyes, J. C. Maize, B. Quearry, M. Vionnet-Fuasset, and E. M. Sternberg. 1990. Scleroderma, fasciitis, and eosinophilia associated with the ingestion of L-tryptophane. $N$. Engl. J. Med. 322:874-881.

14. Medsger, T. A. 1990. Tryptophane-induced eosinophilia-myalgia syndrome. N. Engl. J. Med. 322:926-929.

15. Madara, J. L., and J. S. Trier. 1982. Structure and permeability of goblet cell tight junctions in rat small intestine. J. Membr. Biol. 66:145-157.
16. Madara, J. L., R. Moore, and S. Carlson. 1987. Alteration of intestinal tight junction structure and permeability by cytoskeletal contraction. Am. J. Physiol. 253:C854-C861.

17. Rodewald, R., S. B. Newman, and M. J. Karnovsky. 1976. Contraction of isolated brush borders from the intestinal epithelium. J. Cell Biol. 70:541-545.

18. Keller, T. C. S., and M. S. Mooseker. 1982. $\mathrm{Ca}^{+2}$-calmodulin dependent phosphorylation of myosin, and its role in brush border contraction in vitro. $J$. Cell Biol. 95:943-959.

19. Madara, J. L., D. Barenberg, and S. Carlson. 1986. Effects of cytochalasin D on occluding junctions of intestinal absorptive cells: further evidence that the cytoskeleton may influence paracellular permeability. J. Cell Biol. 97:21252135.

20. Spencer, R. P., and A. H. Samily. 1960. Intestinal transport of L-tryptophane in vivo: inhibition by high concentrations. Am. J. Physiol. 199:1033-1036.

21. Munck, B. G., and S. N. Rasmussen. 1975. Characteristics of rat jejunal transport of tryptophane. Biochim. Biophys. Acta. 389:261-280.

22. Cooke, H. J., and A. R. Cooke. 1982. Effect of tryptophane on transport properties of newborn rabbit jejunum. Am. J. Physiol. 242:G308-G312.

23. Cooke, H. J., L. Pfankuche, and A. R. Cooke. 1980. Tryptophane transport by isolated newborn rabbit jejunum. Am. J. Physiol. 239:G306-G310.

24. Steinart, P. M., and R. K. H. Liem. 1990. Intermediate filament dynamics. Cell. 60:521-523.

25. Tiechberg, S., R. A. Wapnir, M. Zdanowicz, B. Roberts, H. daCosta Ribeiro, and F. Lifshitz. 1989. Morphologic and functional alterations in absorptive cells during L-tryptophane induced inhibition of net sodium and fluid absorption in the rat ileum. Lab. Invest. 60:88-94.

26. Munro, H. N., and M. C. Crim. 1978. The proteins and amino acids. In Modern Nutrition in Health and Disease. 6th ed. R. S. Goodhart and M. E. Shils, editors. Lea \& Febiger, Philadelphia. 88.

27. Belongia, E. A., C. W. Hedberg, G. J. Gleich, K. E. White, A. N. Mayeno, D. A. Loegering, S. L. Dunnette, P. L. Pirie, K. L. MacDonald, and M. T. Osterholm. 1990. An investigation of the cause of the eosinophilia-myalgia syndrome associated with tryptophane use. N. Engl. J. Med. 323:357-365.

28. Heyman, M. B. 1989. Food sensitivity and eosinophilic gastroenteropath ies. In Gastrointestinal Disease. 4th ed. M. Sleisenger and J. Fordtran, editors. W. B. Saunders Company, Philadelphia. 118 